\title{
1 Modelling the population-level protection conferred by COVID-19 vaccination
}

3 Pranesh Padmanabhan ${ }^{1, *}$, Rajat Desikan ${ }^{2, \ddagger}$, Narendra M. Dixit ${ }^{2,3, *}$

$5{ }^{1}$ Clem Jones Centre for Ageing Dementia Research, Queensland Brain Institute, The University of Queensland,

6 Brisbane, Australia 4072

$7 \quad{ }^{2}$ Department of Chemical Engineering, Indian Institute of Science, Bangalore, India 560012

$8 \quad{ }^{3}$ Centre for Biosystems Science and Engineering, Indian Institute of Science, Bangalore, India 560012

$9 \quad{ }^{\ddagger}$ Current Address: Certara QSP, Certara UK Limited, Sheffield, UK

11 *Correspondence:

12 Pranesh Padmanabhan, Narendra M. Dixit

13 Email: p.padmanabhan@uq.edu.au; narendra@iisc.ac.in

15 One sentence summary:

16 Viremic control by the spectrum of neutralizing antibodies elicited by vaccination determines COVID-19 17 vaccine efficacies.

\section{Manuscript details:}

20 Abstract: 125 words; Text: 2500 words; Figures: 4; References: 58 (30 in main text)

21 Supplementary Materials: Tables: 3; Figures: 5 
medRxiv preprint doi: https://doi.org/10.1101/2021.03.16.21253742; this version posted March 20, 2021. The copyright holder for this preprint (which was not certified by peer review) is the author/funder, who has granted medRxiv a license to display the preprint in perpetuity.

All rights reserved. No reuse allowed without permission.

22 Although severe acute respiratory syndrome coronavirus 2 (SARS-CoV-2) vaccines work 23 predominantly by eliciting neutralizing antibodies (NAbs), how the protection they confer 24 depends on the NAb response to vaccination is unclear. Here, we collated and analysed in 25 vitro dose-response curves of $>70$ NAbs and constructed a landscape defining the 26 spectrum of neutralization efficiencies of NAbs elicited. We mimicked responses of 27 individuals by sampling NAb subsets of known sizes from the landscape and found that 28 they recapitulated responses of convalescent patients. Combining individual responses with a mathematical model of within-host SARS-CoV-2 infection post-vaccination, we predicted how the population-level protection conferred would increase with the NAb 31 response to vaccination. Our predictions captured the outcomes of vaccination trials. Our 32 formalism may help optimize vaccination protocols, given limited vaccine availability. Approved SARS-CoV-2 vaccines have shown remarkable but varying efficacies in

35 clinical trials, reducing the incidence of symptomatic infections by $62-96 \%$ (1-4). The 36 protection has been found to be predominantly due to NAbs elicited by the vaccines; cellular 37 immunity appeared to play a secondary role $(1,2)$. The $\mathrm{NAb}$ response elicited by primary 38 SARS-CoV-2 infection is diverse, spanning >1000-fold variation in Ab titres and in vitro 39 neutralization efficiencies across individuals $(5,6)$, and appears not to correlate with disease 40 severity (7). NAb titres following vaccination were comparable to or even lower at times than 41 those from convalescent patients $(1,2,8)$. The protection accorded by the vaccines is thus 42 surprising. It is possible, based on animal studies (9), that lower NAb titres are protective at the 43 time of challenge than post infection. Knowledge of how the level of protection depends on the 
$\mathrm{NAb}$ titres and their neutralization efficiencies is lacking. This knowledge gap hinders rational

optimization of vaccination protocols, which is important today given limited vaccine supplies

(10). Here, we developed a mathematical model that quantitatively predicts the population-

level protection conferred by vaccines as a function of the NAb responses they elicit.

A major challenge to describing the effects of vaccination is the diversity of the NAb

responses elicited; no formalism exists to predict the diversity or its effects on protection. We

50 addressed this challenge by adapting the classic idea of shape space, which has aided

51 quantification of the immune repertoire (11), for characterizing NAbs. Accordingly, we sought

52 features, also termed shape parameters, of the NAbs that would predict their neutralization

53 efficiencies. Numerous studies have isolated individual NAbs from patients and assessed their

54 neutralization efficiencies in vitro, with the aim of developing NAbs for therapeutic

55 applications. We compiled dose-response curves (DRCs) of $>70$ NAbs thus isolated and fit

56 them using the standard sigmoidal function as well as the median-effect equation (12)

57 (materials and methods, fig. S1, table S1). The equations fit the data well (Fig. 1A, and figs.

$\mathrm{S} 2$ and S3), indicating that two parameters, the $50 \%$ inhibitory concentration, $I C_{50}$, and the

slope, $m$, of the DRC, were sufficient to characterize the neutralization efficiency of the NAbs

(Fig. $1 \mathrm{~A}$ and table S1). The best-fit $I C_{50}$ and $m$ varied widely across NAbs (Fig. 1B). $I C_{50}$

61 ranged from $\sim 10^{-3} \mu \mathrm{g} / \mathrm{ml}$ to $\sim 140 \mu \mathrm{g} / \mathrm{ml}$ (Fig. 1B), in close agreement with reported estimates,

62 giving us confidence in the fits (fig. S4A and table S1). $m$, the importance of which has been 

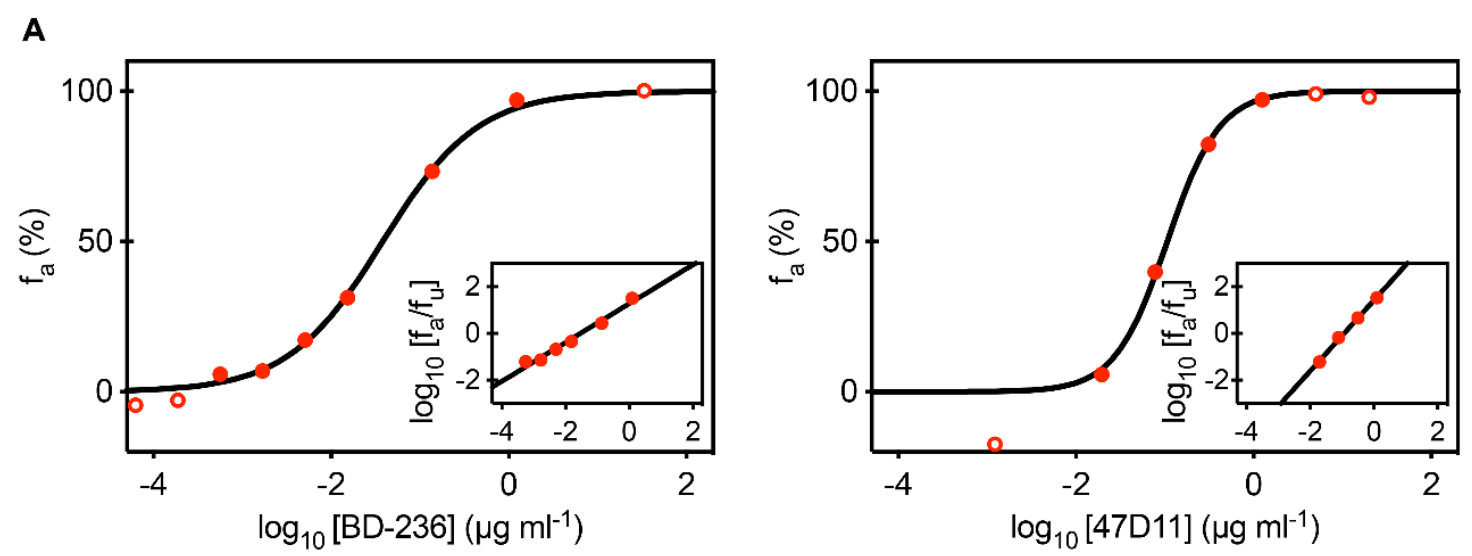

B

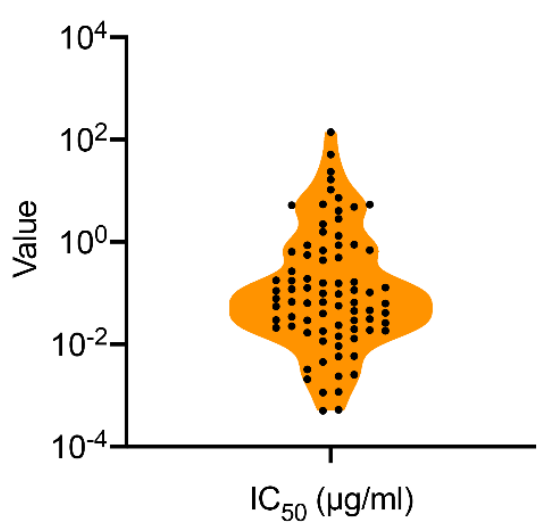

C

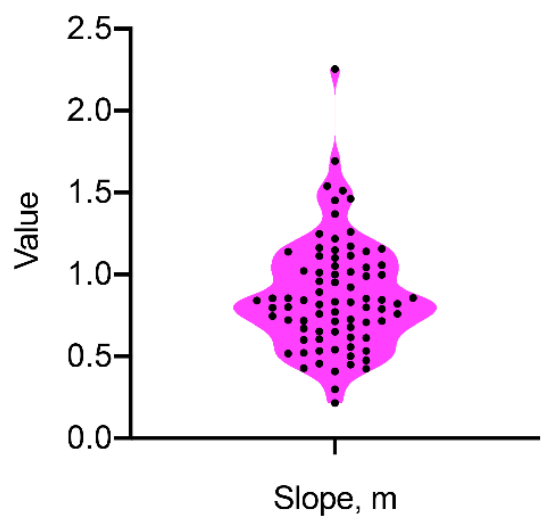

Figure 1. Analysis of dose-response curves of SARS-CoV-2 NAbs. (A) Fits (lines) of the standard sigmoidal equation and the median-effect equation (inset) to published experimental data (circles) of the fraction of infection events blocked, $f_{u}$, as a function of NAb concentration, shown for two NAbs, BD-236 (left) and 47D11 (right). Experimental data points with $1 \%<f_{u}$ $<99 \%$ (filled circles) were considered for parameter estimation. Fits for the remaining NAbs are in figs. S2 and S3. The best-fit estimates of (B) $I C_{50}$ and (C) $m$ for all the NAbs analysed.

to a particular pseudotyped virus construct or backbone used (fig. S4, B and C), the cell line used (fig. S4, D and E), or assay conditions, which could vary across studies (fig. S4, F and G). The variability was thus intrinsic to the NAbs, indicating the spectrum of NAbs elicited. Furthermore, akin to HIV-1 antibodies (12), the variations in $I C_{50}$ and $m$ of the SARS-CoV-2 NAbs appeared independent. For instance, the NAbs BD-361 and REGN10954 had similar $I C_{50}$ 1 (both $\sim 0.04 \mu \mathrm{g} / \mathrm{ml})$, but vastly different $m(\sim 0.7$ and $\sim 1.5$, respectively), whereas the NAbs CC12.3 and 515-5 had vastly different $I C_{50}(\sim 0.02 \mu \mathrm{g} / \mathrm{ml}$ and $1.6 \mu \mathrm{g} / \mathrm{ml}$, respectively), but 
83 similar $m$ (both $\sim 1$ ). $I C_{50}$ and $m$ were thus not only sufficient but also necessary for quantifying

84 the neutralization efficiencies of NAbs. We therefore employed $I C_{50}$ and $m$ as the required

85 shape parameters. Plotting the NAbs on an $I C_{50}-m$ plot, we identified the NAb shape space

(Fig. 2), which, because of its two-dimensional nature, we termed the 'landscape of SARS-

CoV-2 NAbs'.

A

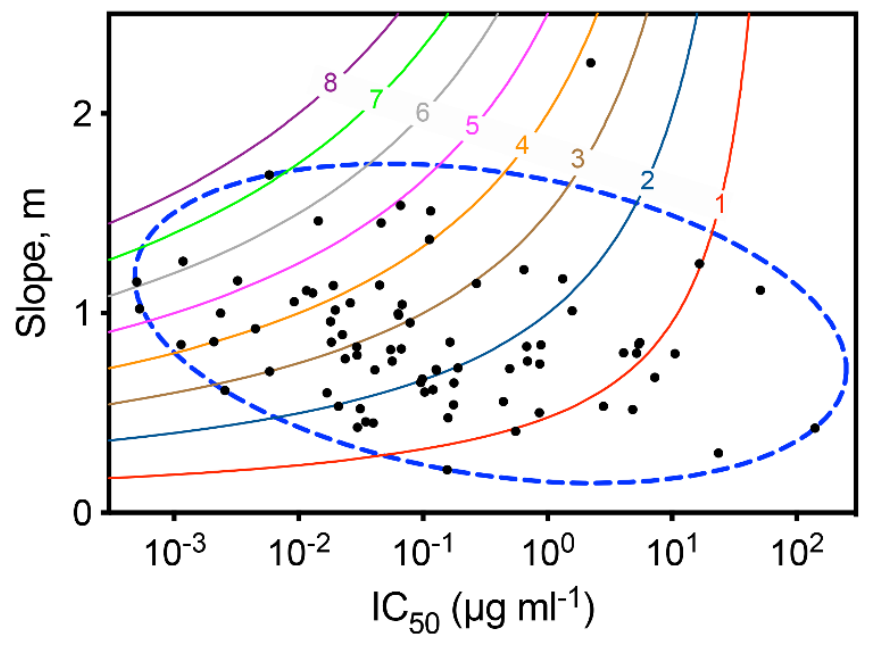

B

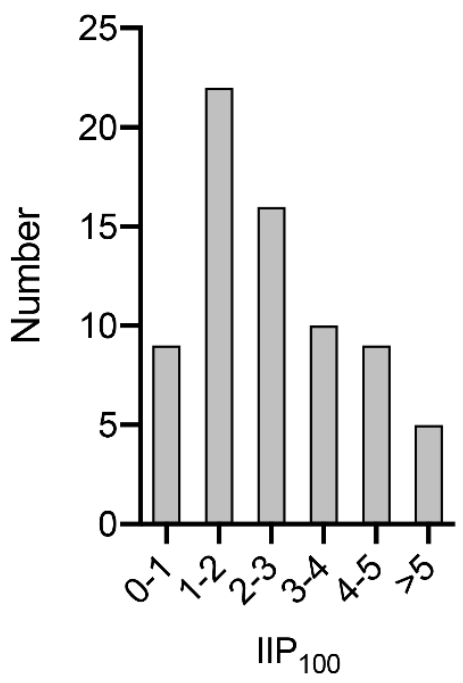

Figure 2. The landscape of SARS-CoV-2 NAbs. (A) SARS-CoV-2 NAbs analysed in Fig. 1 depicted on an $I C_{50}-m$ plot. Each dot represents a NAb. 8 NAbs that have multiple neutralisation curves reported are represented multiple times (table S1). Solid lines are loci of points corresponding to fixed IIP values computed at $100 \mu \mathrm{g} / \mathrm{ml}$. The ellipse (blue dashed line) circumscribes the landscape of SARS-CoV-2 NAbs elicited. (B) The distribution of IIP 100 values of NAbs. Average $I I P_{100}$ values are used for the 8 NAbs mentioned above.

The landscape contains potent NAbs, with low $I C_{50}$ and high $m$, as well as weak NAbs, with the opposite traits. To compare the NAbs, we employed the instantaneous inhibitory potential $(I I P)$, a composite metric of $I C_{50}$ and $m(12-14) . I I P_{D}$ represents the $\log _{10}$ decline in viral load in a single round infection assay due to the NAb present at concentration $D$. Thus, the higher is the $I I P_{D}$, the more potent is the NAb at concentration $D$. NAbs displayed a wide distribution of $I I P_{100}$ values (Fig. $2 \mathrm{~B}$ and table $\mathrm{S} 1$ ): We found that 5 NAbs had the highest $I I P_{100}$ 


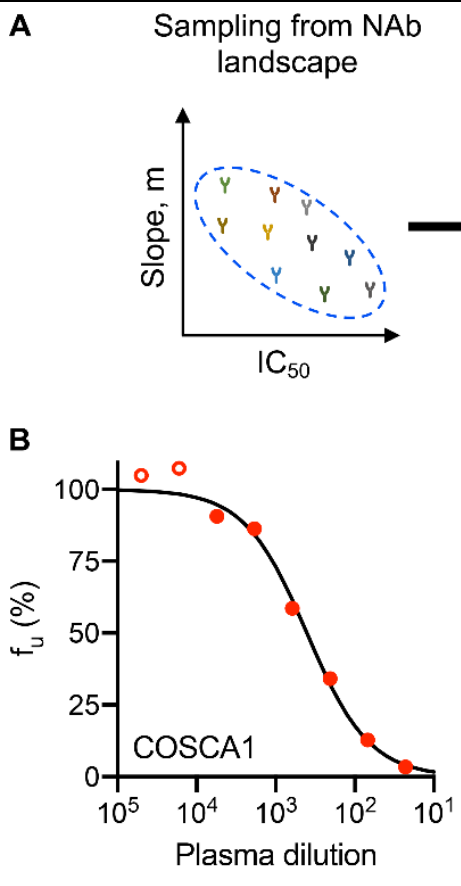

C

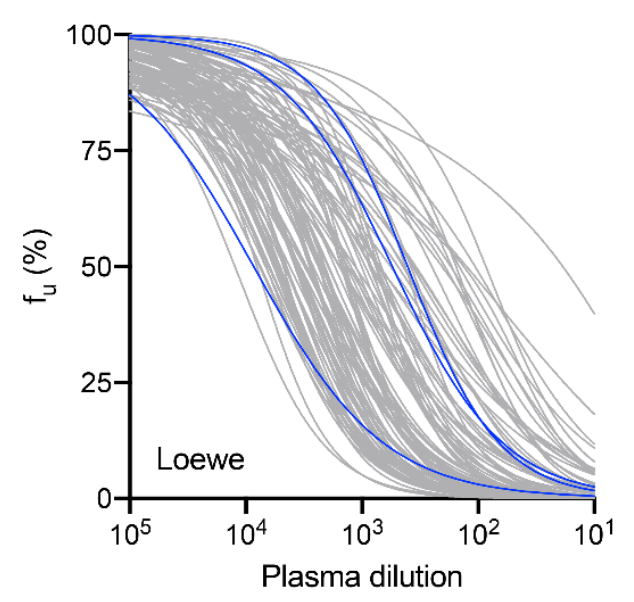

In silico NAb neutralisation
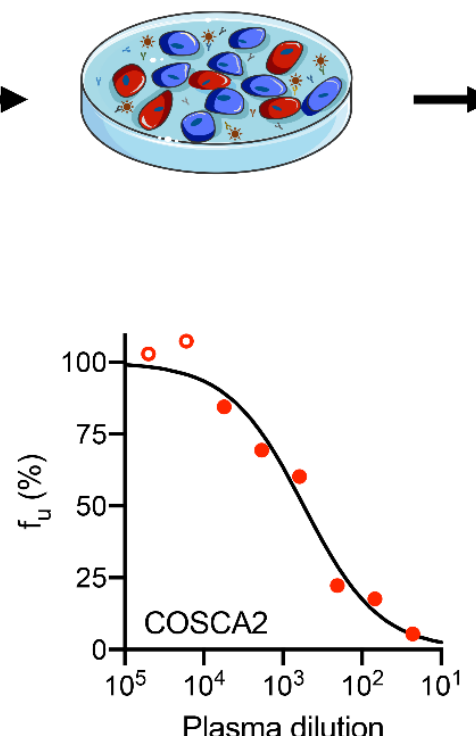

Plasma dilution
Simulated dilution

curve

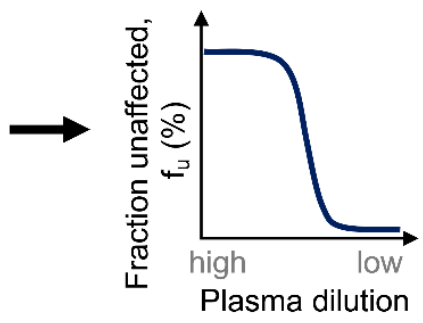

103
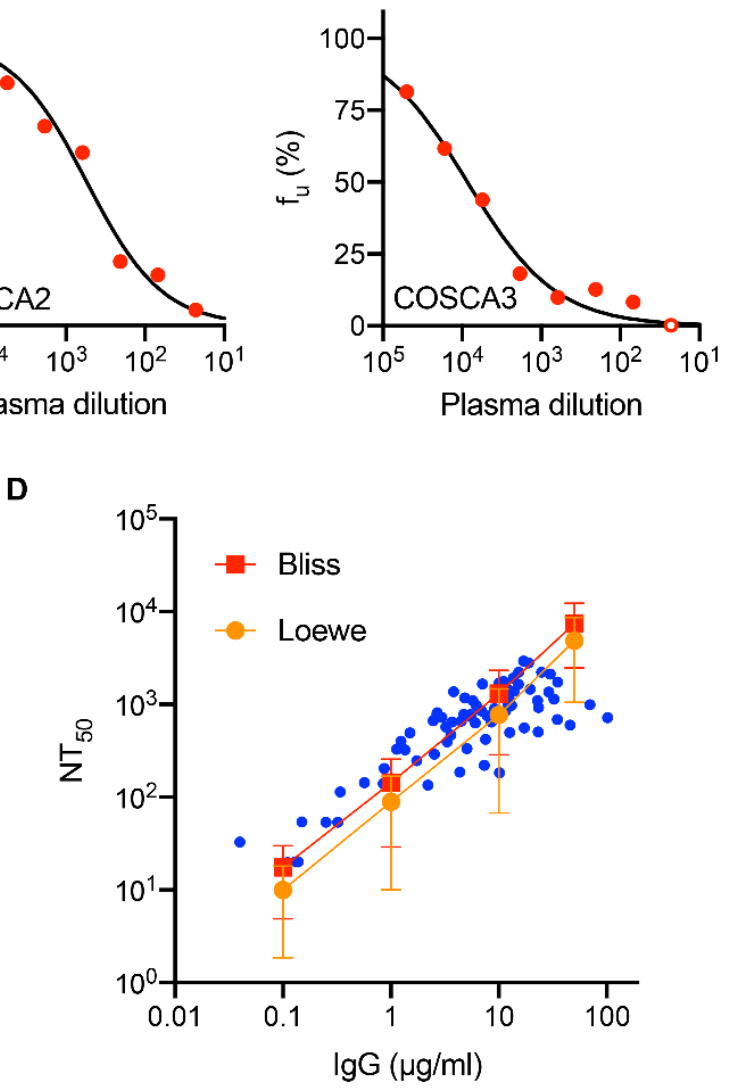

Figure 3. NAb landscape and patient responses. (A) Schematic of the procedure to predict plasma dilution curves. We represent an individual's plasma by a sample of NAbs from the landscape. We predict the fraction of infection events unaffected by NAbs, $f_{u}$, at a given plasma dilution in in vitro pseudovirus neutralization assays and repeat this at different dilutions to obtain the dilution curve. (B) Representative plasma dilution curves obtained as fits (lines) of the equation $f_{u}=\frac{()^{n}}{()^{n}+\left(N T_{50}\right)^{n}}$ to reported data (circles) from three patients (15), where $n$ is the Hill coefficient, $\gamma$ is the plasma dilution and $N T_{50}$ is the half-maximal inhibitory plasma neutralizing titre. Experimental data points with $1 \%<f_{u}<99 \%$ (filled circles) were considered for parameter estimation. (C) Predictions (lines) of plasma dilution curves. We assumed ten NAbs per patient. Blue lines are fits shown in B. $D_{0}=30 \mu \mathrm{g} / \mathrm{ml}$. (D) Half-maximal inhibitory plasma neutralizing titre, $N T_{50}$, as a function of total NAb concentration. Blues circles are reported estimates from convalescent patients. Red squares and orange circles are the mean of $N T_{50}$ values predicted from 100 virtual patients at each NAb concentration using Bliss independence and Loewe additivity, respectively. The error bars are standard deviations. 
values, $>5$, and 9 had the least, $<1(D=100 \mu \mathrm{g} / \mathrm{mL})$ (Fig. 2B and table S1). This distribution

of $I I P_{100}$ values demonstrated further the wide spectrum of neutralization efficiencies of NAbs.

The landscape established bounds on the neutralization efficiencies of the NAbs elicited.

We reasoned next that the diversity of the NAb responses across individuals would arise from

the way NAbs are sampled from the landscape. Although a large number of NAbs can be

isolated from individuals, studies of convalescent patient plasma $(5,6,16-18)$ as well as on

NAb epitope profiling (19) have argued that the NAb response of an individual can be attributed

to a small subset of 5-10 distinct NAbs. Furthermore, while some epitopes on the SARS-CoV-

2 spike protein, S, are targeted more than others by NAbs, the collection of NAbs produced

differs substantially across individuals (20). We therefore assumed that the response elicited

by an infected individual would be a small, random subset of the landscape. We analysed DRCs

of NAbs isolated from individual patients and found that they indeed constituted such random

subsets in the landscape (fig. S5). Accordingly, we sampled random combinations of 10 NAbs

each, each combination representing the response of an individual. We let NAb concentrations

vary across individuals, to mimic the observed variation of the NAb titres (16-18). We

quantified the neutralization efficiency of the NAb response by simulating standard plasma dilution assays (materials and methods, Fig. 3A). We let the NAbs exhibit Bliss independence

or Loewe additivity, the former representing NAbs targeting distinct, non-occluding epitopes

and the latter the same or occluding epitopes (21). Our simulations recapitulated the dilution

curves associated with patient plasma (Fig. 3, B and C). The values of $N T_{50}$, the dilution at

which the neutralization efficiency of the plasma decreases by $50 \%$, were in agreement with 
at low NAb titres and Loewe additivity at high titres. This is expected because at low titres, the

NAbs are unlikely to interact with each other and would thus follow Bliss independence,

whereas at high titres, they may compete for binding sites on $\mathrm{S}$ or occlude each other and thus

exhibit Loewe additivity (21). At any NAb titre, there existed substantial variation in $N T_{50}$,

attributed to the random combinations of NAbs sampled. The variation, however, was

outweighed by the overall rise of $N T_{50}$ with the NAb titre, consistent with patient data (Fig.

3D). For instance, the $N T_{50}$ was $17 \pm 13$ at the $\mathrm{IgG}$ titre of $0.1 \mu \mathrm{g} / \mathrm{ml}$ and $1300 \pm 1000$ at $10 \mu \mathrm{g} / \mathrm{ml}$.

Sampling from the NAb landscape thus successfully recapitulated patient responses. We were

able to describe the diversity of the NAb responses elicited across patients. Armed with this

description, we examined next the protection accorded by vaccines in clinical trials.

Following vaccination, NAb titres rise and are expected to remain stable (or decay

slowly) over weeks to months (22), protecting individuals who might get exposed to the virus

during this period. Individuals were assumed to be protected if they did not report symptomatic

infection; loss of protection involved symptoms and a positive result on a nucleic acid

amplification test $(1,2)$. Protection with NAbs is expected not to be sterilizing, as suggested

by animal studies (9); NAbs help suppress the peak in viremia, thereby reducing symptoms,

and facilitate more rapid clearance of the infection. If the peak is sufficiently suppressed, no

symptoms may result, as is the case with the $\sim 40 \%$ of natural infections that remain

asymptomatic (7). Here, we assumed that an individual would be detected as symptomatically

infected if the viral load rose above a threshold during the infection.

To estimate the peak viral load, we developed a mathematical model of the early time

161 course of the infection, where the viral load typically rises, attains a peak, and declines (23), 

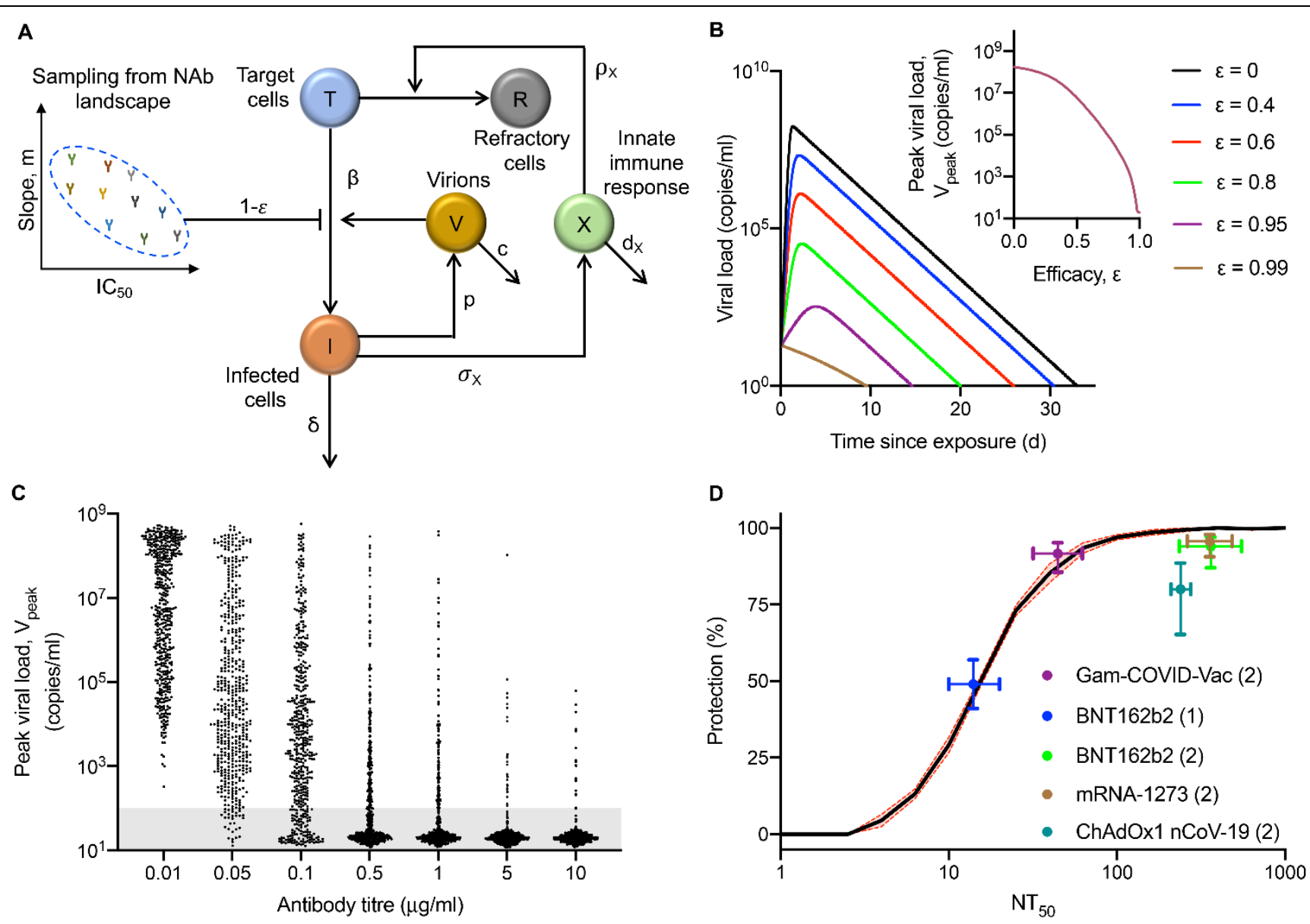

163 Figure 4. SARS-CoV-2 dynamics and protection post-vaccination. (A) Schematic of the model of within-host SARS-CoV-2 dynamics post-vaccination depicting the interactions between target cells, $T$, infected cells, $I$, refractory cells, $R$, virions, $V$, innate immune response, $X$, and pre-existing NAbs, sampled from the landscape. (B) Predictions of viral load in nonvaccinated (black line) and vaccinated (coloured lines) individuals with different fixed efficacies of NAbs indicated. Inset: Predicted peak viral load at different efficacies. (C) Predictions of peak viral load at different NAb titres. Each dot represents a patient. (D) Model predictions of the relationship between mean protection and $N T_{50}$ (solid line) compared with data from vaccination trial (symbols). The number of doses of the vaccine administered is mentioned in brackets. The error bars (dashed lines) in the protection curve are the standard deviation from 5 realizations of in silico patient populations. The data from the trials used is summarized in table S3. The model equations and simulation procedure are described in materials and methods. 
NAbs generated following vaccination would exist at the start of infection and neutralize free

viruses, effectively reducing viral infectivity. The greater the reduction in infectivity, the lower

the peak viral load (Fig. 4B, $\varepsilon>0$ ). Significant de novo NAb production post-infection typically

occurs after the peak in viremia (7). We therefore considered pre-existing NAbs as responsible

for protection and assumed their titres not to vary substantially during the course of the

infection, given the typically short course of the infection and the much longer durability of the

$\mathrm{NAb}$ response to vaccination (22). (Our model is not applicable to natural infection before

vaccination; no models are currently capable of correctly describing NAb responses following

primary infection.) We let the pre-existing NAbs be drawn as random subsets from the

landscape, as we did above. The NAbs neutralized free viruses with an efficiency that we

estimated using Loewe additivity between the individual NAbs (Fig. 4). NAb titres in the lung

airways are expected to be similar to those in the blood given the close coupling between the

lungs and the circulatory system (7). We simulated a virtual patient population of 3500

individuals, on the order of the number of individuals infected in the placebo arms of clinical

trials. The individuals all had distinct viral dynamics parameters drawn from known ranges

(table S2), to mimic interpatient variability in addition to the variability arising from NAb

sampling from the landscape. Our model predicted wide variability in the peak viral load (Fig.

4C). At low pre-existing NAb concentrations $(0.01 \mu \mathrm{g} / \mathrm{mL})$, indicative of the scenario without

vaccination, the predicted peak viral load ranged from $\sim 10^{3}$ to $10^{9}$ copies $/ \mathrm{ml}$, consistent with

the range in symptomatic individuals (26). The peaks declined as NAb titres increased. The

200 limit of detection is $\sim 10^{2}$ copies/ml (27), which we set as the threshold for symptomatic 
infection that would be detected in trials. The fraction of individuals with peaks below detection

would indicate the level of protection due to the vaccine.

To quantify the mean level of protection and test it against data from clinical trials, we

used viral dynamics parameters representative of symptomatic infections $(24,25)$ (table S2)

and simulated the dynamics in 5 cohorts of 2000 infected individuals each. Vaccination studies

report the $N T_{50}$ values of the NAb responses elicited and the associated mean protection level,

207 or efficacy (table S3). We binned the different individuals into narrow $N T_{50}$ bands and

calculated the mean protection in each band. We found that the mean protection was low for

$N T_{50} \sim 1$. It increased in a sigmoidal manner to $50 \%$ at $N T_{50} \sim 20$ and asymptotically reached

$100 \%$ at $N T_{50} \sim 200$. Remarkably, the data for nearly all approved vaccines fell on this

'protection curve', explaining the protection they confer (Fig. 4D). Thus, for instance, a single

dose of the vaccine BNT 162b2 elicited NAbs with $N T_{50}$ of 14 and accorded $49 \%$ protection.

Following two doses, the corresponding values were 361 and 94\%, respectively. These values

as well as those for other vaccines were captured accurately by our model predictions. The only

exception was ChAdOx1 $\mathrm{nCoV}-19$, which had a lower protection than predicted, the reasons

for which remain to be elucidated.

Our study provides the first conceptual, mechanistic and quantitative understanding of the protection conferred by COVID-19 vaccines. Our findings would inform strategies for optimal vaccine deployment. With limited vaccine availability, it would be useful to estimate the protection realizable by a single dose of a prime-boost vaccine, especially in younger, less

221 vulnerable adults (10). Our formalism would enable this estimation: measurements of 
protection curve. Similarly, using measurements of the waning of NAb titres post-vaccination,

224 how the population-level protection due to pre-existing NAbs would fade could be predicted.

225 Protection would then rely on memory B cell responses, which are yet to be fully understood

226 (28), or indicate the need for revaccination. Our study did not consider viral mutations because

227 with 5-10 NAbs active, viral escape from NAb responses is expected to be unlikely $(19,29)$.

228 With the new circulating strains (30), however, the NAb landscape may have to be

229 reconstructed. Future studies may report DRCs of NAbs against the new strains, facilitating

230 such reconstruction.

\section{References}

1. L. R. Baden et al., Efficacy and safety of the mRNA-1273 SARS-CoV-2 vaccine. $N$ Engl J Med 384, 403-416 (2021).

2. F. P. Polack et al., Safety and efficacy of the BNT162b2 mRNA COVID-19 vaccine. $N$ Engl J Med 383, 2603-2615 (2020).

3. M. Voysey et al., Safety and efficacy of the ChAdOx1 nCoV-19 vaccine (AZD1222) against SARS-CoV-2: an interim analysis of four randomised controlled trials in Brazil, South Africa, and the UK. Lancet 397, 99-111 (2021).

4. D. Y. Logunov et al., Safety and efficacy of an rAd26 and rAd5 vector-based heterologous prime-boost COVID-19 vaccine: an interim analysis of a randomised controlled phase 3 trial in Russia. Lancet 397, 671-681 (2021).

5. L. Liu et al., Potent neutralizing antibodies against multiple epitopes on SARS-CoV-2 spike. Nature 584, 450-456 (2020).

6. D. F. Robbiani et al., Convergent antibody responses to SARS-CoV-2 in convalescent individuals. Nature 584, 437-442 (2020).

7. A. Sette, S. Crotty, Adaptive immunity to SARS-CoV-2 and COVID-19. Cell 184, 861880 (2021). 
8. P. M. Folegatti et al., Safety and immunogenicity of the ChAdOx 1 nCoV-19 vaccine against SARS-CoV-2: a preliminary report of a phase 1/2, single-blind, randomised controlled trial. Lancet 396, 467-478 (2020).

9. K. McMahan et al., Correlates of protection against SARS-CoV-2 in rhesus macaques. Nature 590, 630-634 (2021).

10. K. M. Bubar et al., Model-informed COVID-19 vaccine prioritization strategies by age and serostatus. Science 371, 916-921 (2021).

11. A. S. Perelson, G. F. Oster, Theoretical studies of clonal selection: Minimal antibody repertoire size and reliability of self-non-self discrimination. J Theor Biol 81, 645-670 (1979).

12. N. E. Webb, D. C. Montefiori, B. Lee, Dose-response curve slope helps predict therapeutic potency and breadth of HIV broadly neutralizing antibodies. Nat Commun 6, 8443 (2015).

13. P. Padmanabhan, N. M. Dixit, Inhibitors of hepatitis $C$ virus entry may be potent ingredients of optimal drug combinations. Proc Natl Acad Sci U S A 114, E4524-E4526 (2017).

14. B. L. Jilek et al., A quantitative basis for antiretroviral therapy for HIV-1 infection. Nat Med 18, 446-451 (2012).

15. P. J. M. Brouwer et al., Potent neutralizing antibodies from COVID-19 patients define multiple targets of vulnerability. Science 369, 643-650 (2020).

16. B. Isho et al., Persistence of serum and saliva antibody responses to SARS-CoV-2 spike antigens in COVID-19 patients. Sci Immunol 5, eabe5511 (2020).

17. A. S. Iyer et al., Persistence and decay of human antibody responses to the receptor binding domain of SARS-CoV-2 spike protein in COVID-19 patients. Sci Immunol 5, eabe0367 (2020).

18. K. Röltgen et al., Defining the features and duration of antibody responses to SARS-CoV2 infection associated with disease severity and outcome. Sci Immunol 5, eabe0240 (2020).

19. E. Shrock et al., Viral epitope profiling of COVID-19 patients reveals cross-reactivity and correlates of severity. Science 370, eabd4250 (2020).

20. M. Yuan et al., Structural basis of a shared antibody response to SARS-CoV-2. Science 369, 1119-1123 (2020).

21. C. T. Meyer et al., Quantifying drug combination synergy along potency and efficacy axes. Cell Systems 8, 97-108.e116 (2019). 
22. A. T. Widge et al., Durability of responses after SARS-CoV-2 mRNA-1273 vaccination. N Engl J Med 384, 80-82 (2021).

23. R. Wölfel et al., Virological assessment of hospitalized patients with COVID-2019. Nature 581, 465-469 (2020).

24. A. Goyal, E. F. Cardozo-Ojeda, J. T. Schiffer, Potency and timing of antiviral therapy as determinants of duration of SARS-CoV-2 shedding and intensity of inflammatory response. Sci Adv 6, eabc7112 (2020).

25. A. Gonçalves et al., Timing of antiviral treatment initiation is critical to reduce SARSCoV-2 viral load. CPT: Pharmacometrics \& Systems Pharmacology 9, 509-514 (2020).

26. J. Fajnzylber et al., SARS-CoV-2 viral load is associated with increased disease severity and mortality. Nat Commun 11, 5493 (2020).

27. R. Arnaout et al., SARS-CoV2 testing: the limit of detection matters. bioRxiv 10.1101/2020.1106.1102.131144 (2020).

28. R. R. Goel et al., Longitudinal analysis reveals distinct antibody and memory B cell responses in SARS-CoV2 naïve and recovered individuals following mRNA vaccination. medRxiv 10.1101/2021.1103.1103.21252872 (2021).

29. A. Baum et al., Antibody cocktail to SARS-CoV-2 spike protein prevents rapid mutational escape seen with individual antibodies. Science 369, 1014-1018 (2020).

30. T. C. Williams, W. A. Burgers, SARS-CoV-2 evolution and vaccines: cause for concern? Lancet Respiratory Medicine 10.1016/S2213-2600(1021)00075-00078 (2021).

31. X. Chi et al., A neutralizing human antibody binds to the N-terminal domain of the Spike protein of SARS-CoV-2. Science 369, 650-655 (2020).

32. C. Wang et al., A human monoclonal antibody blocking SARS-CoV-2 infection. Nat Commun 11, 2251 (2020).

33. E. Seydoux et al., Analysis of a SARS-CoV-2-infected individual reveals development of potent neutralizing antibodies with limited somatic mutation. Immunity 53, 98-105 e105 (2020).

34. R. Shi et al., A human neutralizing antibody targets the receptor-binding site of SARSCoV-2. Nature 584, 120-124 (2020).

35. A. Z. Wec et al., Broad neutralization of SARS-related viruses by human monoclonal antibodies. Science 369, 731-736 (2020).

36. C. Lei et al., Neutralization of SARS-CoV-2 spike pseudotyped virus by recombinant ACE2-Ig. Nat Commun 11, 2070 (2020). 
37. Z. Lv et al., Structural basis for neutralization of SARS-CoV-2 and SARS-CoV by a potent therapeutic antibody. Science 369, 1505-1509 (2020).

38. S. J. Zost et al., Potently neutralizing and protective human antibodies against SARS-CoV2. Nature 584, 443-449 (2020).

39. B. Ju et al., Human neutralizing antibodies elicited by SARS-CoV-2 infection. Nature 584, 115-119 (2020).

40. Y. Cao et al., Potent neutralizing antibodies against SARS-CoV-2 identified by highthroughput single-cell sequencing of convalescent patients' B cells. Cell 182, 73-84 e16 (2020).

41. J. Hansen et al., Studies in humanized mice and convalescent humans yield a SARS-CoV2 antibody cocktail. Science 369, 1010-1014 (2020).

42. T. F. Rogers et al., Isolation of potent SARS-CoV-2 neutralizing antibodies and protection from disease in a small animal model. Science 369, 956-963 (2020).

43. C. O. Barnes et al., Structures of human antibodies bound to SARS-CoV-2 spike reveal common epitopes and recurrent features of antibodies. Cell 182, 828-842 e816 (2020).

44. D. Pinto et al., Cross-neutralization of SARS-CoV-2 by a human monoclonal SARS-CoV antibody. Nature 583, 290-295 (2020).

45. L. Hanke et al., An alpaca nanobody neutralizes SARS-CoV-2 by blocking receptor interaction. Nat Commun 11, 4420 (2020).

46. X. Chen et al., Human monoclonal antibodies block the binding of SARS-CoV-2 spike protein to angiotensin converting enzyme 2 receptor. Cell Mol Immunol 17, 647-649 (2020).

47. J. Wan et al., Human-IgG-neutralizing monoclonal antibodies block the SARS-CoV-2 infection. Cell Rep 32, 107918 (2020).

48. P. Padmanabhan, N. M. Dixit, Modeling suggests a mechanism of synergy between hepatitis C virus entry inhibitors and drugs of other classes. CPT Pharmacometrics Syst Pharmacol 4, 445-453 (2015).

49. T. C. Chou, Theoretical basis, experimental design, and computerized simulation of synergism and antagonism in drug combination studies. Pharmacol Rev 58, 621-681 (2006).

50. P. Padmanabhan, R. Desikan, N. M. Dixit, Targeting TMPRSS2 and Cathepsin B/L together may be synergistic against SARS-CoV-2 infection. PLoS Comput Biol 16, e1008461 (2020). 
51. V. I. Zarnitsyna et al., Mathematical model reveals the role of memory CD8 $\mathrm{T}$ cell populations in recall responses to Influenza. Front Immunol 7, 165 (2016).

52. R. Ke, C. Zitzmann, R. M. Ribeiro, A. S. Perelson, Kinetics of SARS-CoV-2 infection in the human upper and lower respiratory tracts and their relationship with infectiousness. medRxiv 10.1101/2020.1109.1125.20201772 (2020).

53. D. A. Swan et al., Vaccines that prevent SARS-CoV-2 transmission may prevent or dampen a spring wave of COVID-19 cases and deaths in 2021. medRxiv 10.1101/2020.1112.1113.20248120 (2020).

54. A. S. Perelson, R. Ke, Mechanistic modeling of SARS-CoV-2 and other infectious diseases and the effects of therapeutics. Clin Pharmacol Ther 10.1002/cpt.2160 (2020).

55. N. Dagan et al., BNT162b2 mRNA COVID-19 vaccine in a nationwide mass vaccination setting. N Engl J Med, 10.1056/NEJMoa2101765 (2021).

56. E. E. Walsh et al., Safety and immunogenicity of two RNA-Based COVID-19 vaccine candidates. N Engl J Med 383, 2439-2450 (2020).

57. E. J. Anderson et al., Safety and immunogenicity of SARS-CoV-2 mRNA-1273 vaccine in older adults. N Engl J Med 383, 2427-2438 (2020).

58. M. Voysey et al., Single-dose administration and the influence of the timing of the booster dose on immunogenicity and efficacy of ChAdOx1 nCoV-19 (AZD1222) vaccine: a pooled analysis of four randomised trials. Lancet 397, 881-891 (2021). 
medRxiv preprint doi: https://doi.org/10.1101/2021.03.16.21253742; this version posted March 20, 2021. The copyright holder for this preprint (which was not certified by peer review) is the author/funder, who has granted medRxiv a license to display the preprint in perpetuity.

All rights reserved. No reuse allowed without permission.

\section{Acknowledgements}

369 Funding: This work was supported by the DBT/Wellcome Trust India Alliance Senior

370 Fellowship IA/S/14/1/501307 to NMD. Author contributions: PP: Conceptualization,

371 Investigation, Formal analysis, Writing-original draft, Writing-review \& editing; RD: Formal

372 analysis, Writing-review \& editing. NMD: Conceptualization, Writing-original draft, Writing-

373 review \& editing. Competing interests: The authors declare that no conflicts of interests exist.

374 Data and materials availability: All relevant data are available within the manuscript and

375 supplementary materials. 